\title{
MONTE DO VALE DO OURO 2: OS ENTERRAMENTOS DAS FOSSAS DA PRÉ-HISTÓRIA RECENTE
}

\author{
TÂNIA PEREIRA ${ }^{(1)}$, ANA MARIA SILVA ${ }^{(1),(2),(4)} \&$ ANTÓNIO CARLOS VALERA $^{(5)}$
}

Resumo:

\begin{abstract}
:
\section{Prehistoric pit burials from Monte do Vale do Ouro 2}

In recent years, new data have become available due to the work of rescue archaeology in the South of Portugal (Beja district) that has uncovered a whole new set of Late Prehistoric funerary contexts comprising mainly negative structures (pits, ditches and hypogea). In the archaeological site of Monte do Vale do Ouro 2, two prehistoric pits revealed human bones. The aim of this work is to document and interpret the funerary contexts of these pits based on the analysis of the human bones.

Pit 97 dated to the Chalcolithic revealed the presence of four individuals, one adult female and three non-adults. The mortuary practice of these individuals seems to have involved the exposure to low temperatures confirmed by Fourier Transform Infrared Spectroscopy (FTIC) analysis, which represent a burial practice not yet documented in this region for this period.

In pit 102, dated to the Bronze Age, three adults and one non-adult were exhumed. Among the more relevant data are the evidence of the non-masticatory use of teeth. In two upper lateral incisors, probably antimeres, a profound groove was register near the cement-enamel junction of the lingual part of the teeth suggesting the use of the teeth in daily life, such as processing plant fibers. The non-adult recovered from this pit display a impaction of the second lower premolar, which represents the first archaeological case reported of this condition for the Iberian Bronze Age.
\end{abstract}

Keywords: Multiple burial pits; Signs of fire; Non-masticatory use of teeth; Impaction of lower second premolar.

Received: 16 March, 2017; Accepted: 24 October, 2017

\section{INTRODUÇÃO}

Os trabalhos que têm vindo a ser desenvolvidos no Alentejo, no âmbito da Arqueologia de salvamento, têm gerado novos dados acerca das práticas funerárias das comunidades da Pré-história Recente do Sudoeste Peninsular. Na região, o discurso arqueológico acerca do mundo funerário era baseado no megalitismo, sendo essencialmente com os trabalhos arqueológicos da Barragem de Alqueva, que a realidade se mostrou bastante diferente. Enterramentos em fossa, em fosso e em hipogeus começaram a ser identificados juntamente com complexas práticas de manipulação de restos humanos, o que veio alterar a visão do mundo funerário das comunidades pré-históricas do Alentejo (VALERA 2012).

É nesta realidade que se insere o sítio arqueológico de Monte do Vale do Ouro 2, contendo enterramentos em fossa que datam do Calcolítico e da Idade do Bronze. As deposições humanas neste tipo de estrutura apresentam uma ampla cronologia

\footnotetext{
${ }^{(1)}$ Laboratório de Pré-história, CIAS, Departmento Ciências da Vida, Universidade de Coimbra, 3000-456 Coimbra, Portugal.

(2) UNIARQ - WAPS. Centro de Arqueologia da Universidade de Lisboa.

(3),(4) Laboratório de Antropologia Forense, Centro de Ecologia Funcional (CEF) - Departamento Ciências da Vida, Universidade de Coimbra;

${ }^{(5)}$ Era Arqueologia S.A. / ICArEHB- Universidade do Algarve

Autor correspondente:

Ana Maria Silva

Departmento Ciências da Vida, Universidade de Coimbra, 3000-456 Coimbra (Portugal)

amgsilva@antrop.uc.pt
} 
na região, desde o Neolítico (com exemplos nos Perdigões, Outeiro Alto 2 ou Mina das Azenhas 6; VALERA et al. 2014a; 2014b), passando pelo Calcolítico e apresentando uma maior recorrência durante a Idade do Bronze. Recentemente, e para além das inumações, novos dados têm revelado a presença de depósitos secundários de cremação datados do Calcolítico em fossas, até ao momento apenas identificados no sítio arqueológico dos Perdigões (VALERA et al. 2014a; SILVA et al. 2015).

O objectivo deste trabalho é contribuir para a caracterização das práticas funerárias das comunidades da Pré-história Recente do Sudoeste Peninsular através da análise de duas fossas do sítio de Monte do Vale do Ouro 2. Serão analisadas as condições de deposição e traçado o perfil biológico dos indivíduos exumados, incluindo a análise paleopatológica e o registo de indicadores de stresse fisiológico.

\section{CONTEXTO ARQUEOLÓGICO}

$\mathrm{O}$ sítio arqueológico em estudo localiza-se no Monte do Vale de Ouro, freguesia e concelho de Ferreira do Alentejo, distrito de Beja (Fig. 1). Encontra-se junto à margem esquerda da ribeira do Vale de Ouro, afluente pela margem direita do rio Sado.

Segundo análise da Carta Geológica de Portugal, folha 7, à escala 1:200 000, o sítio de Vale do Ouro 2 desenvolve-se sobre depósitos miocénicos de grés fino e margas calcárias. A área é propensa à formação de terrenos agrícolas, dada a sua riqueza pedológica e hidrológica (OLIVEIRA 1984).

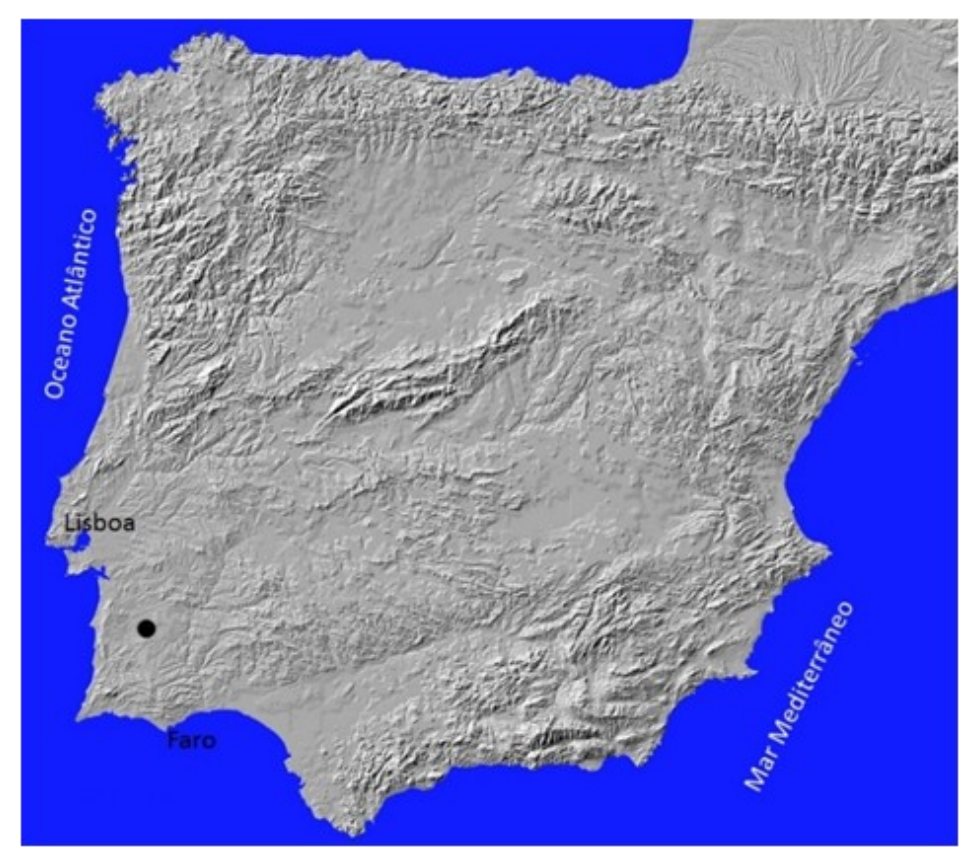

Fig. 1 - Localização do Monte do Vale do Ouro, no mapa da Península Ibérica.

Fig. 1 - Localization of Monte do Vale do Ouro in the Iberian Peninsula.

A escavação do Monte do Vale do Ouro 2 foi realizada no âmbito do projecto de Construção da Subconcessão do Baixo Alentejo (Lanço C), depois da identificação de algumas estruturas durante o acompanhamento da obra. Um total de $187 \mathrm{~m}^{2}$ foram escavados (112 das 114 estruturas identificadas). Das 112 estruturas escavadas, 70 demonstraram pertencer à Pré-história Recente (MORO BERRAQUERO \& FIGUEIREDO 2013). Entre estas, duas fossas revelaram a presença de restos ósseos humanos. A fossa 97, no sector Sudeste, possui paredes convergentes, com 1,65 m de diâmetros de boca, $1,72 \mathrm{~m}$ de base e $0,71 \mathrm{~m}$ de profundidade. No Sector Nordeste, a fossa 102 de paredes convergentes, planta circular, apresenta 1,22 m de boca, 1,24 m na base e $1,16 \mathrm{~m}$ de profundidade.

Localizado no curso da Ribeira do Vale de Ouro, este sítio situa-se próximo do grande com- plexo de recintos de Porto Torrão, o qual é rodeado por vários núcleos com estruturas funerárias, como o Monte do Cardim 6 (tholos), Horta de João da Moura (cinco tholoi), Monte do Carrascal 2 (pelo menos dois tholoi e vários hipogeus) e Monte do Pombal 1 (um tholos) (VALERA 2010).

\section{MATERIAL E MÉTODOS}

A estimativa da idade à morte nos não adultos foi realizada utilizando os restos dentários, através dos métodos de Smith (1991) e AlQahtani et al. (2010). Em relação aos adultos, este parâmetro foi estimado através dos métodos de MacLaughlin (1990) e Schaefer et al. (2009) utilizando, respectivamente, a extremidade esternal da clavícula e a crista ilíaca em fase de fusão. A diagnose sexual baseou-se nas características cranianas (FEREMBACH et al. 
1980; BUIKSTRA \& UBELAKER 1994) e na observação dos ossos longos (WASTERLAIN 2000) e dos ossos do pé (SILVA, 1995).

Alguns destes ossos pareciam demonstrar vestígios de fogo, sendo submetidos a análise de Fourier Transform Infrared Spectroscopy (FTIR), para confirmação. Esta metodologia consiste na medição do Índice de Cristalinidade (IC) e do rácio de Carbonato/Fosfato (C/P) (THOMPSON et al. 2009). Os valores de IC aumentam lentamente no osso dos indivíduos mortos, visto que a sua composição se torna mais ordenada. Apesar de alguns factores diagenéticos poderem aumentar os valores de IC, considera-se que os picos do índice de cristalinidade indicam geralmente osso queimado. Estes valores são combinados com o rácio $\mathrm{C} / \mathrm{P}$ que, geralmente diminuí em ossos queimados, para interpretar as alterações observadas (THOMPSON et al. 2009).

Ao nível da paleopatologia foram pesquisadas lesões degenerativas articulares (CRUBÉZY 1988; ASSIS 2007) e não articulares (MARIOTTI et al. 2004). Também foram pesquisados indicadores de stresse fisiológico como as hipoplasias do esmalte dentário.

O desgaste dentário (SMITH 1984, adaptado por SILVA 1996) e as patologias dentárias pesquisadas foram: as cáries (MOORE \& COORBETT 1971; LUKACS 1989) e os depósitos de tártaro (MARTIN \& SALLER 1956).

\section{RESULTADOS}

\section{Fossa 97}

Durante os trabalhos de campo desta fossa foram identificados três enterramentos no fundo da estrutura (Unidades Estratigráficas [9701], [9702] e [9703]) (Fig. 2), colmatados com a unidade estratigráfica [9700]. O indivíduo 9702 encontrava-se sobre o perímetro N-NE da estrutura e o indivíduo 9703 no perímetro NO da fossa, ambos em decúbito lateral direito, com as pernas flectidas. Já o indivíduo 9701 foi colocado em decúbito dorsal, sobre o enterramento 9702. A presença de um copo carenado e de um bordo de prato junto dos vestígios osteológicos 9702 levou a que se considerasse uma cronologia calcolítica para estes enterramentos (MORO BERRAQUERO \& FigueIREDO 2013).

Posteriormente, em laboratório, identificaram -se na UE 9703 dentes decíduos em formação que não podem pertencer a nenhum dos outros indivíduos da fossa. Encontraram-se ainda, junto ao indivíduo 9703, alguns fragmentos de crânio com reduzida espessura e um côndilo mandibular, junto do esqueleto 9702, que são compatíveis com os dentes decíduos. Assim, o número mínimo de indivíduos (NMI) da fossa 97 é de 4 (1 adulto 9701 e 3 não-adultos).

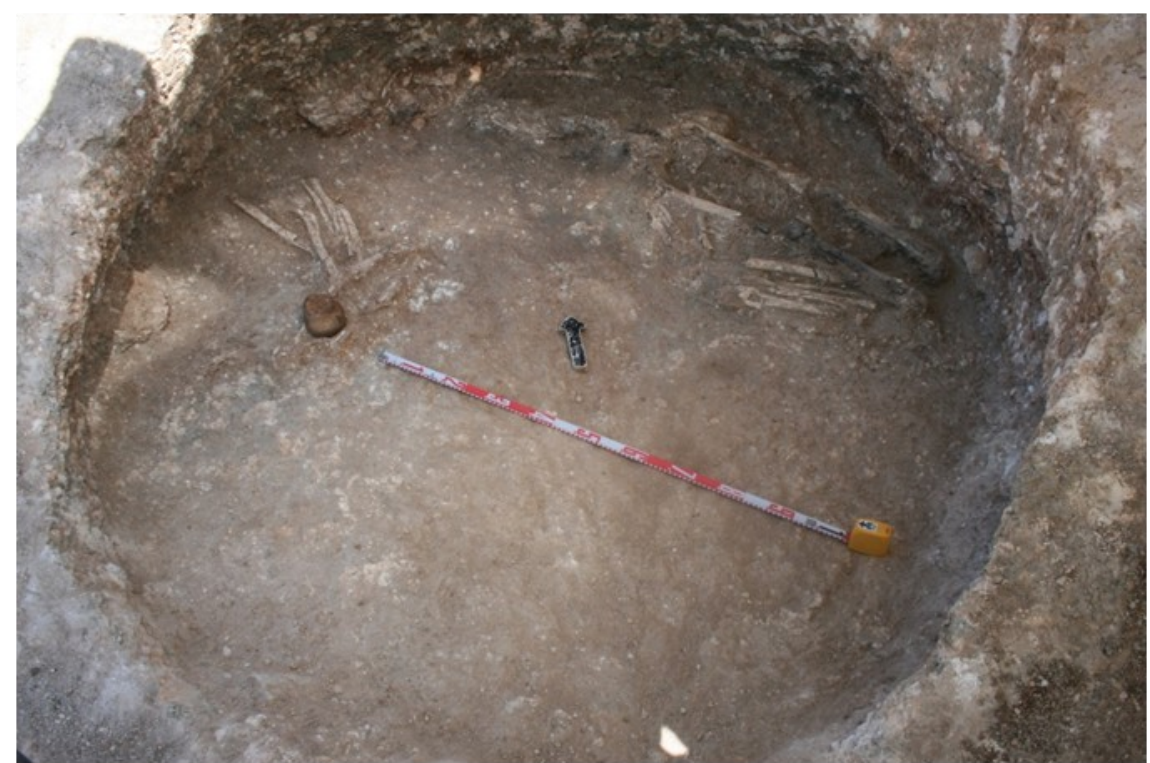

Fig. 2 - Inumações da fossa 97 do sítio do Monte do Vale de Ouro 2: à esquerda, o esqueleto 9703, e à direita, os indivíduos 9701 e 9702 (Moro Berraquero \& Figueiredo 2013).

Fig. 2 - Human remains from pit 97 of Monte do Vale do Ouro 2: on the left, skeleton 9703 and on the right, individuals 9701 and 9702 (Moro Berraquero \& Figueiredo 2013).

Os indivíduos 9701 e 9702 encontravam-se com uma melhor preservação das diáfises dos ossos longos. Já o enterramento 9703 é apenas composto por fragmentos de alguns ossos longos (fémures, tíbias e fíbulas, fragmentos de crânio, mandíbula, dentição permanente inferior em formação).
A diagnose sexual só foi possível no indivíduo 9701, que indicou um indivíduo feminino tendo em conta a gracilidade do côndilo occipital e das diáfises dos ossos longos. Em relação à estimativa da idade à morte, embora o ápex do terceiro molar estivesse fechado, os baixos valores de des- 
gaste dentário indicam que este seria, provavelmente, um adulto jovem. Os restantes indivíduos são não-adultos, tendo em conta a sua dentição. $\mathrm{O}$ enterramento 9702 tinha apenas metade da raiz do terceiro molar formada correspondendo a uma idade entre os 15.6-16.2 anos (SMITH, 1991) e os 16.5-17.5 anos (ALQAHTANI et al. 2010). A dentição permanente em desenvolvimento do esqueleto 9703 revelou uma idade à morte entre os 8-12 a 9.5 -13.5 anos, de acordo com Smith (1991) e AlQahtani et al. (2010). Os dentes decíduos do terceiro indivíduo não adulto mostram que a sua idade se situava entre os 1.5 a 3.8 anos, de acordo com Smith (1991) e cerca de 2.5 anos de acordo com AlQahtani et al. (2010).

Ao nível paleopatológico verificaram-se, sobretudo, patologias dentárias, como lesões cariogénicas, depósitos de tártaro e hipoplasias do esmalte dentário, este último um indicador de stresse fisiológico, todas com frequências, de um modo geral, baixas (Tabela 1).

No indivíduo 9701, foram detectadas duas lesões cariogénicas interproximais $(\mathrm{n}=2 / 26)$, ambas na dentição superior: nos segundo e terceiro molares superiores esquerdos com graus respectivamen- te de, 2 (média) e 1 . Não foram detectados vestígios de tártaro $(\mathrm{n}=0 / 26)$ nem de hipoplasias do esmalte dentário $(n=0 / 25)$. A média do desgaste dentário total é de $1,42(\mathrm{n}=26)$.

No indivíduo não adulto 9702 , o primeiro molar inferior direito apresenta uma cárie interproximal, de grau $1(n=1 / 25)$. A ocorrência de tártaro também revelou uma frequência baixa neste indivíduo $(n=2 / 25)$. Não foram detectadas hipoplasias do esmalte dentário $(n=0 / 25)$ na dentição deste indivíduo.

No esqueleto não adulto 9703, onde apenas a dentição inferior se preservou, não foram registados quaisquer sinais de lesões cariogénicas $(n=0 / 16)$ e as frequências de tártaro e de hipoplasias do esmalte dentário revelaram-se baixas, ambas com 12,6\% $(n=2 / 16)$. Os dentes afectados pelo tártaro foram os dois incisivos centrais inferiores (graus 1 e 3 , respectivamente para o direito $\mathrm{e}$ esquerdo) e pelas hipoplasias do esmalte dentário, os dois caninos inferiores, com uma linha cada.

Apenas o indivíduo 9702, apesar de adolescente, revelou alterações degenerativas articulares, e de grau mínimo: em ambas as extremidades de uma falange distal, e no tarso direito.

Tabela 1 - Frequência de patologias dentárias detectadas nos indivíduos 9701, 9702 e 9703, da fossa 97 de Monte do Vale do Ouro 2.

\begin{tabular}{|c|c|c|c|c|c|c|c|c|c|c|c|c|c|c|c|c|}
\hline & \multicolumn{2}{|c|}{ Cáries } & \multicolumn{2}{|c|}{ Hipoplasias } & \multicolumn{2}{|c|}{ Tártaro } & \multicolumn{2}{|c|}{ Desgaste } & \multicolumn{2}{|c|}{ Cáries } & \multicolumn{2}{|c|}{ Hipoplasias } & \multicolumn{2}{|c|}{ Tártaro } & \multicolumn{2}{|c|}{ Desgaste } \\
\hline & F\% & $\mathbf{N}$ & F \% & $\mathbf{N}$ & F \% & $\mathbf{N}$ & M & $\mathbf{N}$ & F\% & $\mathbf{N}$ & F \% & $\mathbf{N}$ & F \% & $\mathbf{N}$ & $\mathbf{M}$ & $\mathbf{N}$ \\
\hline 9701 & 15,4 & $2 / 13$ & 0 & $0 / 13$ & 0 & $0 / 13$ & 1,67 & 13 & 0 & $0 / 13$ & 0 & $0 / 12$ & 0 & $0 / 13$ & 1,23 & 13 \\
\hline 9702 & 0 & $0 / 11$ & 0 & $0 / 11$ & 0 & $0 / 11$ & 1,27 & 11 & 7,1 & $1 / 14$ & 0 & $0 / 14$ & 14,3 & $2 / 14$ & - & - \\
\hline 9703 & - & - & - & - & - & - & - & - & 0 & $0 / 16$ & 12,5 & $2 / 16$ & 12,5 & $2 / 16$ & - & - \\
\hline
\end{tabular}

Vestígios da acção do fogo foram ainda observados nos esqueletos 9701 e 9702 desta fossa (Fig. 3). No primeiro, estes não se revelaram regulares ao longo de todos os ossos. A região mais afectada foi o esqueleto axial e parte do esqueleto apendicular (clavícula e úmero direitos, parte distal do fémur direito e ambas as tíbias e fíbulas). Foram, ainda, registadas diferentes colorações (negra, azul e branca) ao longo do esqueleto e, nalguns casos, ao longo de um mesmo osso. O indivíduo 9702 apresentava vestígios de fogo nos seus fémures direito e esquerdo, de coloração negra. No início do estudo a origem destas alterações foram questionadas, não sendo possível perceber se realmente seriam sinais de exposição a fogo ou apenas alterações tafonómicas. Uma análise de FTIR (Fourier Transform Infrared Spectroscopy) (THOMPSON et al. 2009) foi realizada nos esqueletos visivelmente afectados (9701 e 9702), mas tam- bém no 9703, sem vestígios aparentes. Dos dois primeiros esqueletos foi retirada uma amostra óssea "normal" e uma de coloração negra. Os resultados demonstraram que os ossos de coloração bege continham valores mais elevados do índice de cristalinidade (CI) e valores mais baixos do rácio de carbonato/fosfato $(\mathrm{C} / \mathrm{P})$, um resultado que não é esperado em ossos não queimados. Pelo contrário, as amostras de osso de coloração negra demonstram níveis de CI e C/P que são compatíveis com ossos que estiveram em contacto com o fogo. Analisando os dados, é possível concluir que todos tenham estado expostos a fogos de baixas ou médias temperaturas, e que o contacto com o fogo não tenha sido regular ao longo de todo o esqueleto. A coloração bege nos ossos queimados poderá ser explicada por algum processo de descoloração ocorrida posteriormente através de processos tafonómicos. 

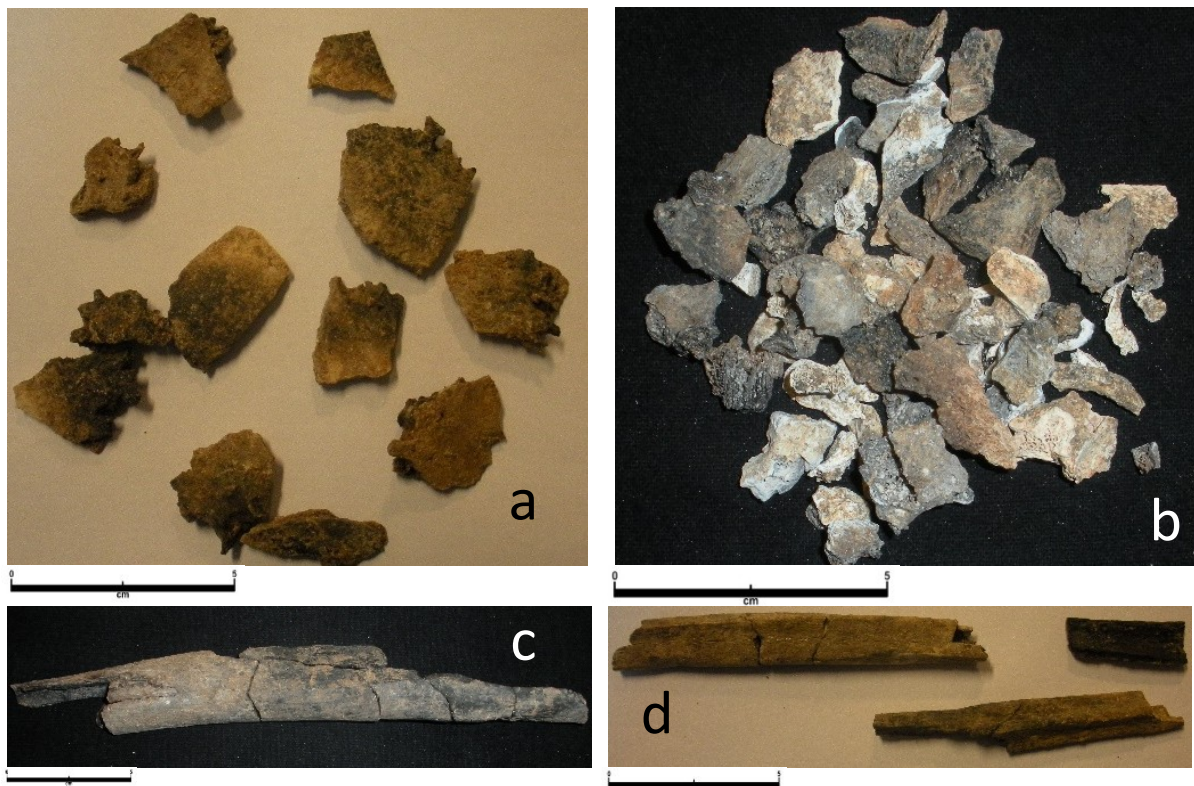

Fig. 3 - Vestígios da acção do fogo nos ossos do esqueleto do indivíduo 9701 exumado da fossa 97 do Monte do Vale de Ouro 2 (a - fragmentos cranianos; b - fragmentos de vértebras; c - parte de diáfise da tíbia; $\mathrm{d}$ - fragmentos de fíbula).

Fig. 3 - Signs of exposure to fire in the bones of skeleton 9701 recovered from pit 97 of Monte do Vale de Ouro 2 (a - cranial fragments; $b$ - vertebral fragments; $c$ - fragments of tibae; $d$-fragments of fibula).

\section{Fossa 102}

Durante os trabalhos de campo foram identificas três fases de inumação nesta estrutura (Fig. 4). Primeiramente foi exumada uma unidade estatrigráfica [10200], com características que se verificavam noutras estruturas de cronologias préhistórica (MORO BERRAQUERO \& FIGUEIREDO 2013), com presença de rochas, [10201]. Esta presença observou-se em maior quantidade por cima da primeira inumação 10202, depositada em decúbito lateral direito, com os membros flectidos. Entre esta e a seguinte inumação 10205, colocada também em decúbito lateral direito, detectou-se uma unidade estratigráfica [10203] com inclusões de caliços. Ao lado da inumação 10205 encontrava -se um depósito de rochas [10204] que foi retirado juntamente com a inumação (MORO BERRAQUERO \& Figueiredo 2013). Após mais duas unidades estratigráficas, [10206] e [10208], encontrava-se o último enterramento 10207, um indivíduo depositado em decúbito lateral esquerdo. Quanto ao material arqueológico desta estrutura, destacam-se uma conta ou pendente de osso cilíndrico, fragmentos cerâmicos de formas abertas com carena, um fragmento de uma taça de perfil em $\mathrm{S}$ e dois bordos simples de formas semifechadas, um dos quais com um mamilo junto ao bordo. Cronologicamente esta estrutura pode enquadrar-se na Idade do Bronze (Moro Berraquero \& Figueiredo 2013).

Ainda que durante os trabalhos de escavação tenham sido identificados apenas três enterramentos nesta fossa (10202, 10205 e 10207), a análise laboratorial detectou a presença de alguns ossos dispersos na UE 10200, elevando o NMI. Nesta unidade estratigráfica encontravam-se alguns fragmentos de fémur e de cúbito, cuja elevada espessu-
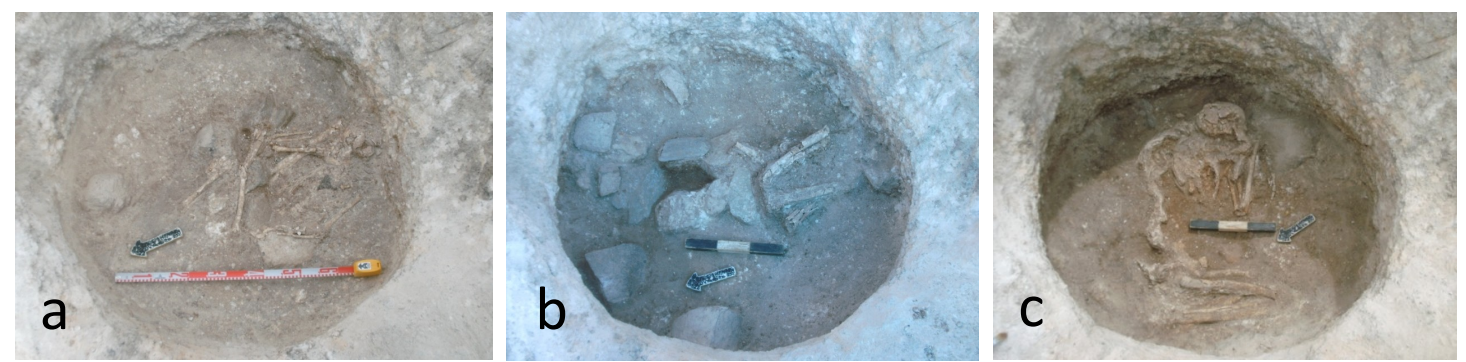

Fig. 4 - Fossa 102 de Monte do Vale do Ouro 2 (a - enterramento 10202; b - enterramento 10205; c - enterramento 10207) (MORO BERRAQUERO \& FIGUEIREDO 2013).

Fig. 4 - Pit 102 from Monte do Vale do Ouro 2 (a - Burial 10202; b - Burial 10205; c - Burial 10207) (Moro Berraquero \& FigueireDO 2013). 
ra do tecido cortical não permite que sejam compatíveis com o esqueleto 10205. Estes restos ósseos também não podem pertencer aos restantes ossos exumados desta fossa porque o esqueleto 10207 tem as diáfises dos ossos longos praticamente inteiras e o esqueleto 10202 pertence a um não-adulto. Similarmente um pré-molar inicialmente registado como pertencente ao indivíduo 10207 , não pode pertencer a este ou a nenhum dos enterramentos inicialmente identificados, pelo que é possível que se relacione com o esqueleto da UE 10200. Assim, o NMI da fossa 102 é de 4 (3 adultos e 1 nãoadulto 10202) (Fig.5).

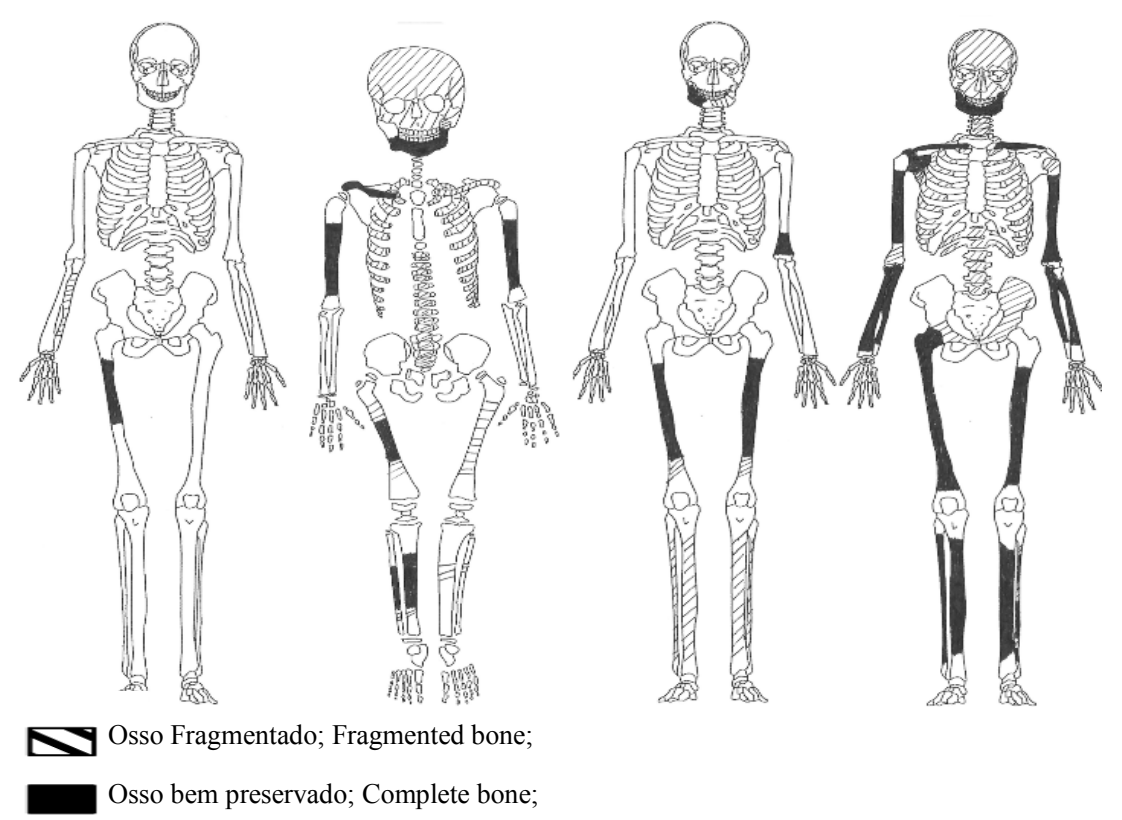

Fig. 5 - Representação dos ossos presentes nos esqueletos 10200, 10202 (não adulto), 10205 e 10207, na estrutura 102 de Monte do Vale do Ouro 2.

Fig. 5 - Skeletal preservation of burials 10200, 10202 (non - adult), 10205 and 10207 from pit burial 102 from Monte do Vale do Ouro 2.

Os vestígios osteológicos encontravam-se, de um modo geral, muito fragmentados, principalmente no esqueleto axial. O indivíduo 10207, comparativamente com os outros indivíduos da fossa, é o melhor preservado, com as diáfises dos ossos longos praticamente inteiras, embora as extremidades quase todas destruídas.

A diagnose sexual recorrendo a métodos métricos apenas foi possível no indivíduo 10207 já que nos indivíduos 10200 e 10205 apenas foi possível avaliar a robustez/gracilidade do esqueleto. Relativamente ao indivíduo 10207, a observação das apófises mastóides e da proeminência mental sugerem o sexo feminino, tal como o diâmetro vertical da cabeça do fémur (39 mm).

A estimativa da idade à morte foi realizada em três indivíduos. No enterramento 10202 foi possível estimar a idade à morte através da dentição. Os dentes permanentes revelaram uma idade à morte entre os 5 - 6,8 anos (SMITH, 1991) e 6,5 7,5 anos (ALQAHTANI et al., 2010). Relativamente à fusão das epífises, foi possível observar que o apéx do dens do axis ainda não se encontrava fundido ( $<12$ anos), ao contrário da junção dento neural (> 4-6 anos). O indivíduo 10207, apresenta o terceiro molar inferior direito com metade do apex fechado, o que corresponde a uma idade entre os 19,1 - 19,4 anos (Smith, 1991) e $19-22,5$ anos (ALQAHTANI et al. 2010), enquanto a fusão da crista ilíaca sugere uma idade à morte superior a 21 anos. Do indivíduo 10205 apenas se observou a perda antemortem de dentes e a redução da espessura de tecido cortical, indicadores que sugerem um adulto não muito jovem.

Ao nível das patologias articulares, foram detectadas lesões nos esqueletos 10200 (extremidade proximal do cúbito direito, de grau mínimo) e 10207 (extremidades proximais do rádio e ulna direitos detectaram-se vestígios de artrose de grau 1). Relativamente às enteses, registaram-se alterações nos mesmos indivíduos: 10200 (lesões osteofíticas de grau médio, nas regiões de inserção do M. supinator e M. brachialis nos fragmentos de cúbito direito) e 10207 (alterações osteolíticas de grau mínimo no rádio direito, na inserção do $\mathrm{M}$. biceps brachii e também nos fragmentos de costelas e vértebras observáveis).

No registo das patologias dentárias, no indivíduo 10202 nenhuma lesão cariogénica $(n=0 / 10)$ ou depósitos de tártaro $(n=0 / 10)$ foram registados na 
dentição permanente superior. Na dentição inferior permanente não se registaram quaisquer cáries $(\mathrm{n}=0 / 5)$, depósitos de tártaro $(\mathrm{n}=0 / 5)$ ou hipoplasias do esmalte dentário $(\mathrm{n}=0 / 5)$. O mesmo aconteceu na dentição decídua, superior $(\mathrm{n}=0 / 5)$ e inferior $(n=0 / 2)$, que não revelaram nenhum sinal destas patologias (Tabela 2). Já no indivíduo 10207 observou-se uma cárie interproximal, de grau médio (2) no primeiro molar superior direito e no segundo pré -molar e primeiro molar inferiores esquerdos, respectivamente, de graus 1 e $2(n=3 / 22)$. Não foram registados quaisquer sinais de tártaro ou hipoplasias do esmalte dentário. Apenas se registaram alguns vestígios de tártaro em alguns dentes da dentição inferior $(37,5 \%$; $n=6 / 16)$, de grau mínimo no primeiro e segundo molares direitos, incisivo lateral direito e canino esquerdo, e de grau 2 nos incisivos centrais esquerdo e direito. A média total do desgaste dentário é de $1,78(\mathrm{n}=23)$ (Tabela 2).

No indivíduo não adulto 10202 não ocorreu a erupção do segundo pré-molar inferior esquerdo, encontrando-se a região do osso alveolar fechado, apesar do segundo molar decíduo já não estar presente. A mandíbula foi alvo de um Raio X realizado num mamógrafo digital (Mammograph General Electrics, senographeDMR, voltagem: $26 \mathrm{kV}$; exposição: $18 \mathrm{mAs}$ ), que mostrou o $2^{\circ}$ pré-molar "retido" pelo primeiro molar definitivo devido à sua inclinação anómala na cripta (Fig. 6).

Ao indivíduo 10205 parecem pertencer dois dentes incisivos laterais superiores tendo em conta o seu avançado nível de desgaste. A mandíbula deste indivíduo revela também perda antemortem dos dentes posteriores. Os incisivos laterais possuem desgaste na parte lingual e oclusal do dente e um sulco na junção cimento-esmalte (Fig. 7).

É ainda importante mencionar que é visível um achatamento na parte acromial da clavícula esquerda do indivíduo 10207, a região de inserção do músculo deltóide de natureza desconhecida (Fig. 8).

Tabela 2 - Frequência de patologias dentárias na dentição superior permanente nos indivíduos 10202 e 10207 do Monte do Vale do Ouro 2.

\begin{tabular}{|c|c|c|c|c|c|c|c|c|c|c|c|c|c|c|c|c|}
\hline & \multicolumn{8}{|c|}{ Dentição superior } & \multicolumn{8}{|c|}{ Dentição Inferior } \\
\hline & \multicolumn{2}{|c|}{ Cáries } & \multicolumn{2}{|c|}{ Hipoplasias } & \multicolumn{2}{|c|}{ Tártaro } & \multicolumn{2}{|c|}{ Desgaste } & \multicolumn{2}{|c|}{ Cáries } & \multicolumn{2}{|c|}{ Hipoplasias } & \multicolumn{2}{|c|}{ Tártaro } & \multicolumn{2}{|c|}{ Desgaste } \\
\hline & F\% & $\mathbf{N}$ & F \% & $\mathbf{N}$ & F \% & $\mathbf{N}$ & $\mathbf{M}$ & $\mathbf{N}$ & $\mathbf{F} \%$ & $\mathbf{N}$ & F \% & $\mathbf{N}$ & F \% & $\mathbf{N}$ & M & $\mathbf{N}$ \\
\hline 10202 & 0 & $0 / 10$ & 0 & $0 / 10$ & 0 & $0 / 10$ & - & - & 0 & $0 / 5$ & 0 & $0 / 5$ & 0 & $0 / 5$ & - & - \\
\hline 10207 & 16,7 & $1 / 6$ & 0 & $0 / 7$ & 0 & $0 / 7$ & 2,14 & 7 & 12,5 & $2 / 16$ & 0 & $0 / 16$ & 37,5 & $6 / 16$ & 1,63 & 16 \\
\hline
\end{tabular}
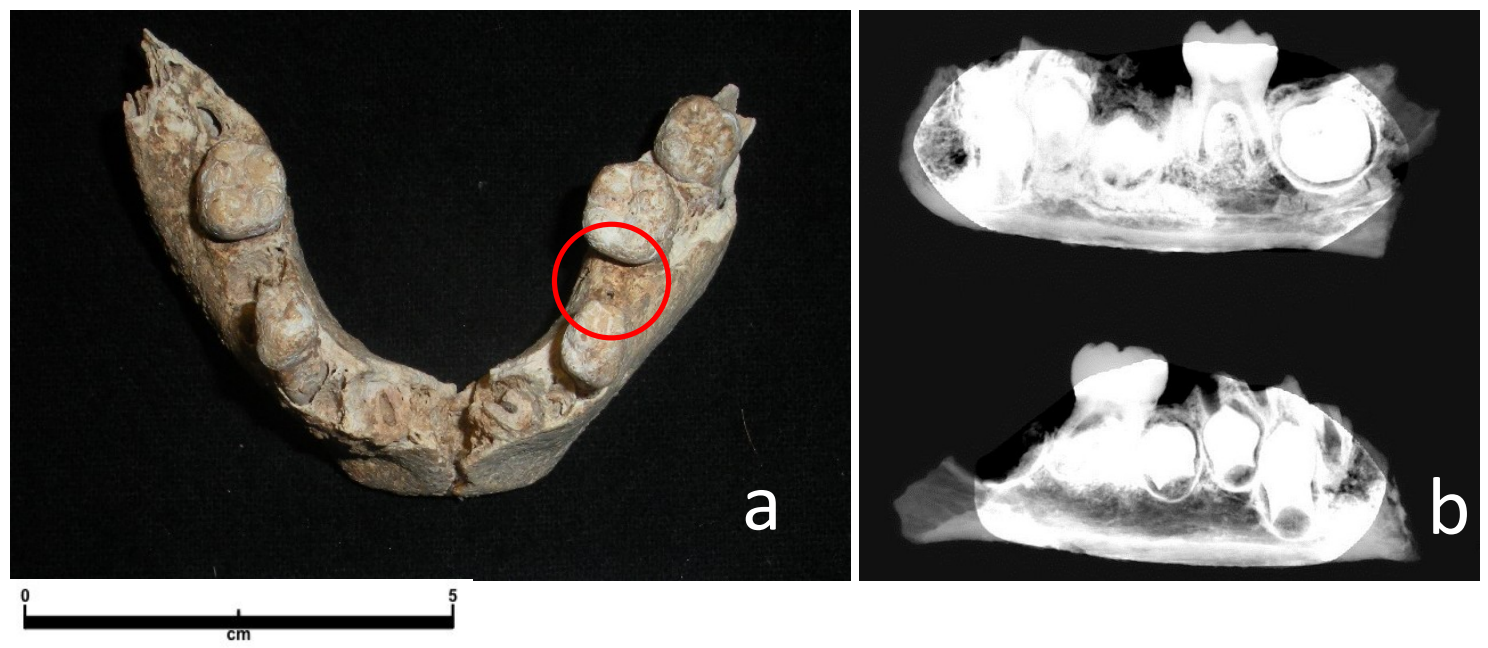

Fig. 6 - a - Mandíbula do indivíduo 10202 do Monte do Vale de Ouro 2, onde é visível a ausência de erupção do $2^{\circ}$ pré-molar esquerdo; b - O raio-X da mandíbula do mesmo indivíduo: em cima, o lado direito; em baixo, o esquerdo, onde é visível a presença do $2^{\circ}$ pré-molar inferior.

Fig. 6 - a - Mandible of the individual 10202 exhumed from Monte do Vale de Ouro 2: Note that the second lower premolar has not erupted; b - X-ray from mandible 10202; top: right side; bottom, left side were the presence of the lower second premolar is confirmed. 


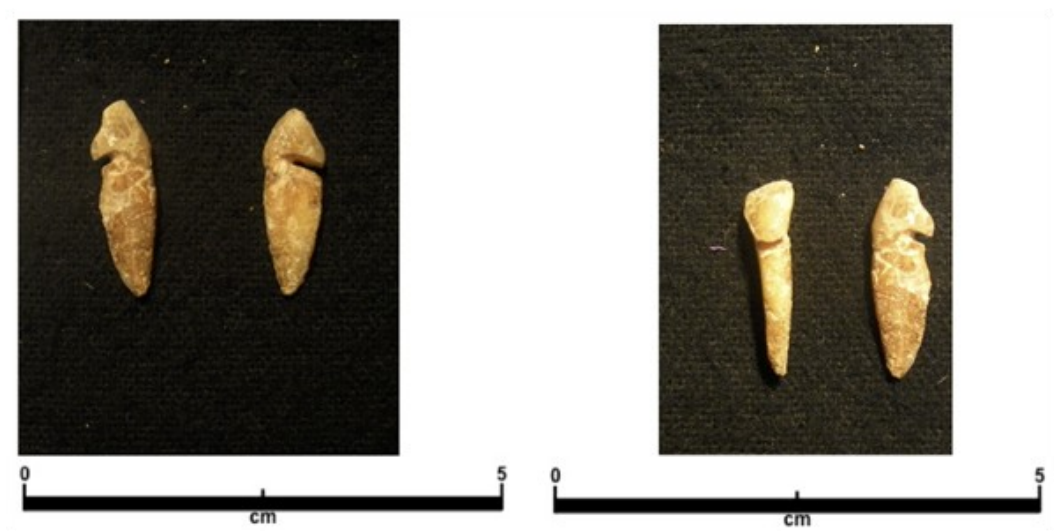

Fig. 7. Incisivos laterais superiores esquerdo e direito recuperados da UE 10200, possivelmente pertencentes ao indivíduo 10205, com evidências de uso não mastigatório (fossa 102, Monte do Vale do Ouro 2).

Fig. 7. Upper left and right central incisors recovered from UE 10200 possibly belonging to individual 10205, displaying evidence of non-masticatory use of teeth (Pit 102 from Monte do Vale do Ouro 2).

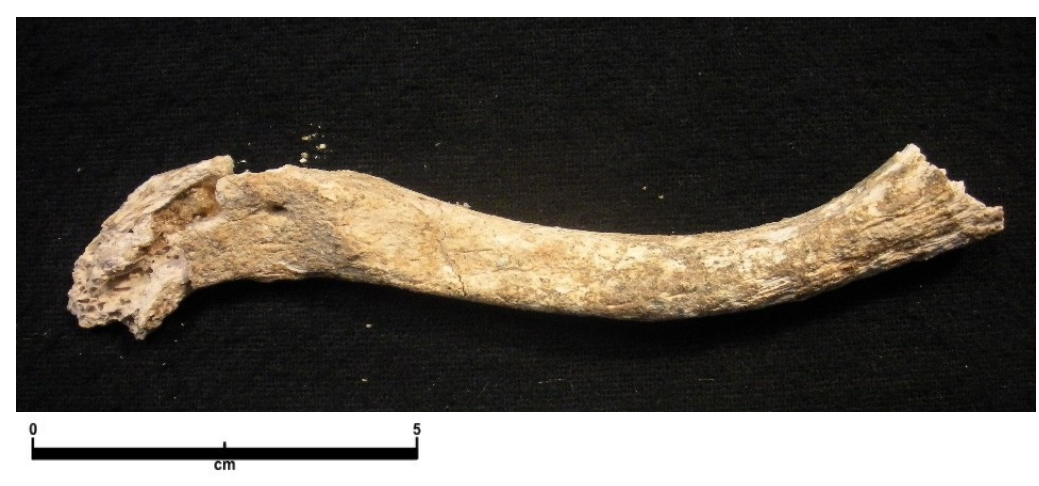

Fig. 8. Clavícula esquerda com achatamento inferior na parte acromial (Indivíduo 10207, Monte do Vale do Ouro 2).

Fig. 8. Left clavicle from individual 10207 from pit 102 of Monte do Vale do Ouro 2 with inferior flatness of the acromial end.

\section{DISCUSSÃO}

As duas fossas analisadas neste trabalho continham enterramentos múltiplos correspondendo a um mínimo de 4 indivíduos: a fossa 97 com 1 indivíduo adulto e 3 não adultos e a fossa 102 , com 1 não-adulto e 3 adultos. Relativamente à diagnose sexual, apenas foi possível sugerir o sexo feminino para indivíduo adulto da fossa 102 (10207). Estes dados confirmam a existência de adultos e não adultos em ambas as fossas.

As posições de inumação passíveis de análise encontravam-se em decúbito lateral direito, posição flectida, à excepção do esqueleto 9701 que se encontrava em decúbito dorsal, com as pernas estendidas. As orientações dos enterramentos foram mais variáveis: O-E, NO-SE e SE-NO.

$\mathrm{Na}$ fossa 97 destacam-se os vestígios de acção do fogo em vários dos ossos dos indivíduos 9701, 9702 e 9703. Análises de FTIR, envolvendo os índices de $\mathrm{CI}$ e o rácio $\mathrm{C} / \mathrm{P}$, revelam que os resultados obtidos para as amostras retiradas destes indivíduos enquadram-se na possibilidade de estes terem estado sujeitos a fogo de baixa intensidade, embora de forma inconsistente. Estas evidências não representam casos de cremação como acontece, por exemplo, nas fossas 16 e no ambiente 1 do sítio arqueológico dos Perdigões (SILVA et al., 2015). Várias são as interpretações de vestígios de fogo encontrados em contextos funerários da Préhistória Recente da Península Ibérica: consequência de fogos de higienização ocorridos à superfície (ARMENDARIZ 1990); fogo como meio de iluminação no momento da inumação (ROJO GUERRA et al. 2002); fogo representando carácter ritual e simbólico (MATUTANO 2013). Em relação à amostra de Monte do Vale do Ouro 2, como as evidências são de exposição a baixas temperaturas, não é possível confirmar se a combustão dos ossos do esqueleto depositado estratigraficamente mais acima terá afectado os outros, ou se ambos terão sido sujeitos a fogo. Pode ainda colocar-se a hipótese de que a 
distinta posição de inumação do indivíduo 9701 (decúbito dorsal) esteja relacionado com a exposição ao fogo. É, também, possível verificar que na unidade estratigráfica superior existiam alguns carvões dispersos, sem nenhuma grande concentração que indicasse práticas relacionadas com o fogo à superfície da estrutura. A queima parcial de ossos, semelhante à que aqui se encontra, tem sido registada nalgumas grutas da Península Ibérica como Avenc dels Dos Forats (Valência), com uma coloração que variava entre o negro e vários tons de cinzento (GARCÍA PUCHOL et al. 2010), e El Rebollosillo e Juan Barbero (região de Madrid) (AliagA ALMELA 2008). Mas também nalguns monumentos megalíticos. Contudo, estes contextos diferem muito do encontrado em Monte do Vale do Ouro 2, já que por serem túmulos colectivos de enterramentos sucessivos e não simultâneos, dando origem a reduções contínuas que se assemelham com deposições secundárias, ao contrário de Monte do Vale do Ouro 2 onde se verificam enterramentos primários com preservação das conexões anatómicas.

Relativamente à análise paleopatológica, foram observadas poucas lesões degenerativas articulares nos restos ósseos recuperados das duas fossas de Monte do Vale do Ouro 2. Contudo, devem ser tidas em conta as alterações tafonómicas que, muitas vezes, destruíram as regiões articulares, não permitindo a sua observação. Na fossa 97 apenas o indivíduo 9702, apesar de adolescente, revelou algumas alterações articulares nomeadamente numa falange distal (extremidade proximal e distal) e no tarso direito. Na fossa 102 detectaram-se lesões de grau mínimo em dois esqueletos na região articular do cotovelo, sendo que um dos esqueletos foi afectado tanto no cúbito como no rádio. A articulação do cotovelo tende a ser bastante afectada em colecções arqueológicas, principalmente na articulação úmero-radial, ao contrário do que sucede nas populações recentes (ORTNER 2003).

As entesopatias são uma patologia da região da entese causada por factores mecânicos, metabólicos, entre outros, e que, em colecções arqueológicas se acredita que possam fornecer informações acerca do modo e qualidade de vida das populações, principalmente em relação à actividade física dos indivíduos (MARIOTTI et al. 2004). No entanto, a fragmentação da amostra, o número reduzido de indivíduos e a dificuldade no estabelecimento de diagnoses sexuais, dificulta inferências acerca desta patologia na amostra em estudo. Assim, apenas se registaram este tipo de alterações não articulares em dois indivíduos da fossa 102 (lesões osteofíticas nos fragmentos de cúbito direito de um indivíduo de sexo indeterminado e alterações osteolíticas no rádio direito, nos fragmentos de costelas e nas vértebras observáveis, de outro indivíduo de sexo feminino).

Ao nível da patologia dentária a cárie é uma das mais frequentes na dentição das populações do passado (SILVA 2012). Contudo, parece ser apenas a partir da Idade Média que a frequência de cáries começa a ser mais elevada (principalmente as cáries oclusais), sendo que o consumo de cereais utilizados por comunidades agrícolas parece relacionar-se, sobretudo, com as cáries na raiz e na junção do cimento-esmalte (LARSEN 1997; SILVA 2012). Assim, nesta amostra apesar do reduzido número de indivíduos, verificou-se a presença de cáries nos dois indivíduos adultos com dentição observável, embora a frequência registada de dentes afectados por indivíduo tenha sido reduzida $(7,69 \% \mathrm{n}=2 / 26$, no indivíduo 9701 e $13,6 \%$ $n=3 / 22$, no indivíduo 10207). Nos não-adultos apenas um dos três indivíduos com dentição observável foi afectado, registado-se um dos dentes com cárie $(4 \% \mathrm{~N}=1 / 25$, no indivíduo 9702). As cáries registadas foram todas interproximais, em dentes molares, entre os graus 1 (pequena) e 2 (média).

$\mathrm{O}$ tártaro, ou placa mineralizada, é frequentemente associada a populações com pouca higiene oral. Em amostras como esta, muito fragmentada e afectada por processos tafonómicos, o seu registo dificulta-se já que depois do enterramento muitas vezes o tártaro desaparece. Nos indivíduos aqui estudados, apenas se registaram depósitos de tártaro com baixa frequência $(26,1 \% n=6 / 23)$ num indivíduo adulto (10207) de dois observáveis, e nos dois não adultos $(9702-8 \% \mathrm{n}=2 / 25 ; 9703-12,6 \%$ $\mathrm{n}=2 / 16$ ), sendo os dentes afectados incisivos, caninos e molares, com graus de 1 a 3 .

As hipoplasias do esmalte dentário são defeitos no esmalte, sob a forma de linha ou pits, que ocorrem devido a factores de stresse fisiológico que afectaram o indivíduo. Os dentes mais afectados são geralmente os incisivos centrais superiores e os caninos inferiores (AUFDERHEIDE \& RODRÍGUEZ-MARTÍN 1998). Nesta amostra verificou-se a presença deste tipo de patologia apenas num indivíduo não-adulto ( $9703 ; n=2 / 16)$ dos três com dentição observável, cujos dentes afectados foram os caninos inferiores.

Apesar do desgaste dentário não ser uma patologia, o seu registo é essencial já que a sua ocorrência se relaciona com factores morfológicos dos dentes, com outras patologias (como defeitos no esmalte), com a dieta dos indivíduos e com comportamentos não mastigatórios (SILVA 2002). Nos dois indivíduos adultos das fossas 97 e 102 onde o desgaste foi estudado, este revelou médias baixas $(9701-1,42, n=26 ; 10207-1,78, n=23)$, o que se pode dever também ao facto de, provavelmente, se tratarem de adultos jovens.

Contudo, além destes valores baixos de desgaste registados encontraram-se, ainda, dois dentes com evidências de uso não-mastigatório na fossa 102. O uso dos dentes para outras actividades que não as relacionadas com a alimentação podem fornecer informações acerca de algumas actividades de subsistência das populações do passado, como a 
utilização dos dentes como ferramenta, e do seu comportamento cultural, como o caso da sua higiene (MINOZZI et al. 2003; LORKIEWICZ 2011). Algumas lesões orais foram interpretadas como consequência da utilização de "palitos" em Homo erectus, Neandertais e aborígenes australianos (UBELAKER et al. 1969). Os padrões de desgaste não-mastigatório são variáveis, aparecendo nos dentes posteriores e anteriores, deixando sulcos interproximais ou oclusais (BERMUDEZ DE CASTRO et al. 1997; LORKIEWICZ 2011), possivelmente resultantes de actividades e comportamentos diferentes. Alguns sulcos oclusais e interproximais de dentes posteriores e anteriores têm sido interpretados como consequência de actividades de tecelagem, cestaria, produção de redes, cordas e fibras, sendo os dentes utilizados como uma "terceira mão", produzindo assim os sulcos visíveis nos dentes (LORKIEWICZ 2011). Nos incisivos laterais superiores da fossa 102 de Monte do Vale do Ouro 2 , o sulco observado surge junto à linha cimentoesmalte, no lado lingual, dando a ideia de que algum tipo de fio ou fibra seria continuamente passado na região. $\mathrm{O}$ desgaste mais ténue registado também na parte distal da superfície lingual corrobora esta hipótese. A tecelagem, produção de fios, redes, cestas ou cordas são uma possibilidade neste caso. Contudo, já que os dentes se encontravam soltos, torna-se impossível verificar a existência de mais lesões noutros dentes, não permitindo também a observação da posição dos dentes no maxilar, impedindo de perceber com mais rigor o tipo de movimento que poderá ter dado origem a este tipo de lesão.

De referir ainda alguns casos particulares como o da mandíbula do não-adulto (10202) da fossa 102, com uma idade à morte estimada entre os 5 - 7,5 anos (AlQAHTANi et al., 2010 e SMITH, 1991), cujo alvéolo do segundo molar decíduo esquerdo se encontrava completamente remodelado. A perda prematura do molar decíduo, por razões desconhecidas, pode ter estado na origem desse facto. Em 1962, Fanning registou uma erupção mais rápida dos pré-molares de crianças (entre os 7,5 e 8 anos e os 9 e 9,5 anos) cujos segundos molares decíduos foram extraídos. Porém, nalguns destes casos é possível que o primeiro molar permanente se desloque para mesial, podendo causar a impactação do segundo pré-molar inferior, impedindo a sua erupção (FANNING 1962; POSEN 1965). Isto parece ter acontecido com o indivíduo desta amostra.

Ainda na mesma fossa, no indivíduo adulto jovem 10207, verifica-se o achatamento de uma clavícula na região de inserção do músculo deltóide. Este achatamento pode ser provocado por algum esforço continuado, relacionado com alguma actividade de carácter desconhecido. Contudo, uma variação anatómica não pode ser excluída. Curiosamente, casos semelhantes foram observa- dos num não adulto da Ribeira de S. Domingos 1 (PEREIRA 2014) e na colecção osteológica de Torre Velha 3 (FIDALGO 2014).

\section{CONSIDERAÇÕES FINAIS}

A análise dos vestígios osteológicos das fossas 97 e 102 do Monte do Vale do Ouro 2 insere-se no estudo do mundo funerário do Baixo Alentejo, onde a heterogeneidade de soluções de enterramento tem vindo a ser posta a descoberto com os trabalhos de Arqueologia de Salvamento, revelando alternativas ao tradicional megalitismo que é predominante no Norte e Centro alentejano.

A amostra osteológica aqui estudada, apesar de pequena, revela aspectos dessas soluções alternativas, neste caso a de deposições múltiplas em fossas atribuíveis ao Calcolítico e Idade do Bronze. Por outro lado, a utilização do fogo nas práticas funerárias detectada na Fossa 97 reforça a ideia de que durante o $3^{\circ}$ milénio AC a queima parcial a baixas temperaturas e a cremação terão tido uma importância até há pouco insuspeita nas formas de tratamento do corpo, contribuindo para a diversidade de práticas e manipulações dos restos humanos que tem vindo a ser reconhecida no Calcolítico regional. Por último, a amostra permite ainda engrossar a informação disponível relativamente a perfis demográficos e dados biológicos e patológicos, revelando ainda alguns detalhes relevantes, como o uso de dentes para fins não mastigatórios.

\section{AGRADECIMENTOS}

David Gonçalves pelo apoio nas análises de Fourier Transform Infrared Spectroscopy (FTIC) dos restos ósseos da fossa 97 e ao apoio financeiro do CIAS (PEst-OE/SADG/ UI0283/2013).

\section{BIBLIOGRAFIA}

Aliaga Almela, R. 2008. El mundo funerario calcolítico en la Región de Madrid. CuPAUAM, 34: 23-39.

AlQhitani, S.J.; Hector, M.P. \& LiVersidge, H.M. 2010. Brief communication: the London altlas of human tooth development and eruption, American Journal of Physical Anthropology, 142(3): 481-490.

Armendariz, A. 1990. Las Cuevas Sepulcrales en el País Vasco. Munibe (Antropologia-Arkeologia), 42: 153 -160 .

ASSIS, S.S.D. 2007. A memória dos rios no quotidiano dos homens: contributo de uma série osteológica proveniente de Constância para o conhecimento dos padrões ocupacionais. Dissertação de Mestrado em Evolução Humana Coimbra, Faculdade de Ciências e Tecnologia, Universidade de Coimbra.

Aufderheide, A. \& Rodríguez-Martín, C. 1998. Cambridge encyclopedia of human paleopathology. Cambridge, Cambridge University Press.

Bermudez de Castro, J.M.; Arsuaga, J.L. \& Perez, P.J. 1997. Interproximal Grooving in the Atapuerca-SH Hominid Dentitions. American Journal of Physical Anthropology, 102: 369-376. 
BUIKSTRA, J.\& UBELAKER, D. 1994. Standards for data collection from human skeletal remains. Proceedings of a Seminar at the Filed Museum of Natural History. Fayetteville, Arkansas, Arkansas Archaeological Survey Research Series 44.

CRUBÉZY, E. 1988. Interactions entre facteurs bio-culturels, pathologie et caracteres discrets: exemple d'une population médiéval. Thèse de Doctorat. Montpellier, Université de Montpellier.

FANNING, E. 1962. Effect of extraction of deciduous molars on the formations and eruption of their successors. The Angle Orthodontist, 32 (1): 44-53.

Ferembach, D.; Schwidetzky, I.\& Stloukal, M. 1980. Recommendations for age and sex diagnoses of skeletons. Journal of Human Evolution, 9: 517-549.

FidALGO, D. 2014. Contextos funerários e estudo antropológico dos restos ósseos humanos dos hipogeus de Torre Velha 3 (São Salvador, Serpa): uma aproximação ao estudo das comunidades humanas do Bronze do Sudoeste. Dissertação de Mestrado em Evolução e Biologia Humanas, Departamento de Ciências da Vida da Faculdade de Ciências e Tecnologia da Universidade de Coimbra.

García Puchol, O.; Cotino Villa, F.; Miret Estruch, C.; Pascual Benito, J. L.; McClure, S.B., MoliNA BALAGUER, L.; ALAPONT MARTÍN, L.; CARRIÓN Marco, Y.; Morales, J. V.; Blasco Senabre, J. \& Culleton, B. 2010. Cavidades de uso funerario durante el Neolítico final/Calcolítico en el territorio valenciano: trabajos arqueológicos en Avenc dels Dos Forats o Cova del Monedero (Carcaixent, Valencia), Archivo de Prehistoria Levantina, XXVIII: 139-206.

LORKIEWICZ, W. 2011. Nonalimentary Tooth Use in the Neolithic Population of the Lengyel Culture in Central Poland (4600-4000 BC). American Journal of Physical Anthropology, 144: 538-551.

LUKACS, J.R. 1989. Dental anthropology: methods for reconstruction dietary patterns. In M. Iscan \& $\mathrm{K}$. Kennedy (Eds.), Reconstruction of life from the skeleton. Nova Iorque, Alan R. Liss Inc.: 261-286.

MaCLAUGHLIN, S.M. 1990. Epiphyseal fusion at the sterna end of the clavicle in a modern Portuguese skeletal sample. Antropologia Portuguesa, 8: 59-68.

Mariotti, V.; Facchini, F. \& Belcastro, M. 2004. Enthesopaties - Proposal of a standardized scoring method and applications. Collegium Anthrologicum, 28 (1): 145- 159.

MARTIN, R. \& SALLER, K. 1956. Lehrbuch der Anthropologie 1. Stuttgart, Gustav FisherVerlag.

Matutano, P.V. 2013. Cueva Maturras (Ciudad real, españa): el papel del fuego en un contexto funerario del III Milenio a.C. Saguntum. Papeles del Laboratorio de Arqueología de Valencia, 45: 39-47.

MinozZI, S.; ManZI, G.; Ricci, F.; Lernia S.\& TARLi, S.M.B. 2003. Nonalimentary Tooth Use in Prehistory: An Example From Early Holocene in Central Sahara (Uan Muhuggiag, Tadrart Acacus, Libya). American Journal of Physical Anthropology, 120: 225-232.

MoORE, W.J. \& CORBETT, M.E. 1971. The distribution of dental caries in ancient British populations. I. Anglo -Saxon period. Caries Research, 5: 151-168.
Moro Berraquero, J. \& Figueiredo, A. 2013. Subconcessão da Auto-estrada do Baixo Alentejo - Lanço CRelatório final dos trabalhos arqueológicos de Monte de Vale do Ouro 2. Relatório Omniknos.

OliveirA, J.T. (coord) 1984. Carta geológica de Portugal, na escala 1: 200000 , notícia explicativa da folha 7 Lisboa, Serviços Geológicos de Portugal.

ORTNER, D.J. 2003. Identifications of Pathological Conditions in Human Skeletal Remains. Second Edition. San Diego, Academic Press.

PEREIRA, T. 2014. Enterramentos em fossa no distrito de Beja. Práticas funerárias e estudo dos vestígios osteológicos da Pré-história recente. Dissertação de Mestrado em Evolução e Biologia Humanas, Departamento de Ciências da Vida da Faculdade de Ciências e Tecnologia da Universidade de Coimbra.

PoSEN, A.L. 1965. The effect of premature loss of deciduous molars on premolar eruption. The Angle Orthodontist, 35 (3): 249-252.

Rojo Guerra, M. A.; Kunst, M.\& Palomino, L. 2002. E fuego como procedimiento de clausura en tres tumbas monumentales de la Submeseta Norte. In: M Rojo Guerra \& M. Kunst (Eds.), Sobre el significado del fuego en los rituales funerarios del Neolitico. Valladolid: 21-38.

SChaefer, M.; Black, S. \& SCheuer, L. 2009. Juvenile Osteology: a laboratory and field manual. London, Academic Press.

SiLVA, A.M. 2002. Antropologia funerária e paleobiologia das populações portuguesas (Litorais) do Neolítico Final/ Calcolítico. Dissertação de Doutoramento em Antropologia, Departamento de Antropologia, Universidade de Coimbra.

SiLVA, A.M. 2012. Antropologia funerária e paleobiologia das populações portuguesas (litorais) do Neolitico final/Calcolítico. Fundação Calouste Gulbenkian/ Fundação para a Ciência e Tecnologia.

Silva, A.M.; Leandro, I.; Pereira, D.\& Valera, A.C. 2015. Collective secondary cremation in a Pit Grave: a unique funerary context in Portuguese Chalcolithic burial practices. Journal of Comparative Human Biology, 66(1): 1-14.

SMITH, B.H. 1984. Patterns of molar wear in hunter-gatheres and agriculturalists.American Journal of Physical Anthropology, 63: 39-84.

SMITH, B.H. 1991. Standards of human tooth formation and dental age assessment. In: M. Kelley \& C.S. Larsen (Eds.), Advances in Dental Anthropology, New York, Wiley- Liss: 143-168.

Thompson, T.J.; Gauthier, M.\& Islam, M. 2009. The application of a new method of Fourier Transform Infrared Spectroscopy to the analysis of burned bone. Journal of Archaeological Science, 36: 910914.

Ubelaker D.H.; Phenice, T.W.\& BAss, W.M. 1969. Artificial interproximal grooving of the teeth in American Indians. American Journal of Physical Anthropology, 30 (1): 145-149.

VALERA, A.C. 2010. Gestão da morte no $3^{\circ}$ milénio AC no Porto Torrão (Ferreira do Alentejo): um primeiro contributo para a sua espacialidade. Apontamentos de Arqueologia e Património: 57-62. 
VALERA, A.C. 2012. Ditches, pits and hypogea: new data and new problems in South Portugal Late Neolithic and Chalcolithic funerary practices. In: J. Gibaja, A. Carvalho \& P. Chambon (Eds.), Funerary Practices in the Iberian Peninsula from the Mesolithic to the Chalcolithic. Oxford, Archeopress: 103-112.

Valera, A.C.; Silva, A.M.; Cunha, C. \& Evangelista, L.S. 2014a. "Funerary practices and body manipulations at Neolithic and Chalcolithic Perdigões ditched enclosures (South Portugal). In: A.C.Valera (Coord.), Recent Prehistoric Enclosures and Funerary Practices. Oxford: BAR Internacional Series 2676: 37-57.
VAlera, A.C.; Godinho, R.; ClaVo; E.; BerReQUero, F.J.M. \& FiliPE,V.; SANTOS, H. 2014b. "Um mundo em negativo: fossos, fossas e hipogeus entre o Neolítico Final e a Idade do Bronze na margem esquerda do Guadiana (Brinches, Serpa)". $4^{\circ}$ Colóquio de Arqueologia do Alqueva. O plano de rega (2002-2010), Memórias d'Odiana, $2^{\text {a }}$ Série, 14, Edia/DRCALEN: 55-73.

WASTERLAIN, S. 2000. Morphé: análise das proporções entre os membros, dimorfismo sexual e estatura de uma amostra da Colecção de Esqueletos Identificados do Museu Antropológico da Universidade de Coimbra. Dissertação de Mestrado em Evolução Humana, Faculdade de Ciências e Tecnologia, Universidade de Coimbra. 